\title{
Perspective taking in judgment of relative direction tasks
}

\author{
Danit Geva ${ }^{1} \cdot$ Avishai Henik ${ }^{1}$ \\ Published online: 15 April 2019 \\ (C) The Psychonomic Society, Inc. 2019
}

\begin{abstract}
Mental rotation and visual-spatial perspective taking (VSPT) are two visual-spatial abilities that are highly correlated. Judgment of relative direction (JRD) tasks are commonly used to examine VSPT in conjunction with mental rotation. Moreover, in many studies the task involves switching from an imagined perspective to a sensorimotor perspective at the response phase. The current study employed a JRD task that enabled examination of mental rotation, perspective taking, and perspective switching, separately. In two experiments we found that both perspective taking and perspective switching affected accuracy and response time (RT). Namely, trials with a perspective change larger than $90^{\circ}$ (that required perspective taking) were harder than trials with a change smaller than $90^{\circ}$ (that required mental rotation), and so were trials that required switching from an imagined perspective to a sensorimotor perspective. Importantly, we suggest that VSPT and mental rotation are dissociable.
\end{abstract}

Keywords Mental transformation $\cdot$ Perspective switching $\cdot$ Visual-spatial perspective taking $\cdot$ Mental rotation

\section{Introduction}

Visual-spatial perspective taking (VSPT) is a fundamental component for human survival. Our mind is constrained to a body that can only be in one place at a time at any given moment. Despite that, we are required to recognize and to make judgments about different objects and locations in space, in a world that is constantly changing around us, and in which we constantly change. Moreover, human beings communicate with each other for survival. People experience space differently and have different information about the world at every given moment. A substantial portion of this information is experienced or manipulated mentally in our mind. Mentalizing visual-spatial information is important for maintaining a coherent sense of our environment and for creating a common ground for communication. This is exemplified when one adopts a new perspective. Taking a novel perspective also requires detaching from what we see (i.e., sensorimotor perspective) and moving to a mentalized perspective. The current work examines perspective taking using a new judgment of relative direction (JRD) task - the dots perspective task (DPT). The DPT enabled us to examine mentalizing new

Danit Geva

danitg@post.bgu.ac.il

1 Department of Psychology, Ben-Gurion University of the Negev, P.O.B. 653, Beer-Sheva, Israel perspectives as well as switching between perspectives, using the congruency between two parts of the task.

\section{Challenges in visual-spatial perspective taking (VSPT) measurement}

The first psychometric test of VSPT ability was applied on children using Piaget's three-mountain task (Piaget \& Inhelder, 1956, 1966/1971). In this task, participants were shown a model of three mountains on a table. A doll was positioned next to the table and participants were asked to make judgments about how the scene looked from the doll's perspective. Later, additional VSPT tests were developed and applied on adults. The most common test was the GuilfordZimmerman spatial orientation test (i.e., GZSOT) (Guilford \& Zimmerman, 1948). In this test, participants are provided with a specific point of view of a landscape and asked to infer a boat's position.

A main limitation of psychometric tests is that they often mix several processes and do not isolate the precise mental process of interest (Carroll, 1993). Visual-spatial tests can be solved by many different strategies, thus they measure unpure factors (Hegarty, 2010; Just \& Carpenter, 1985; Kyllonen, Lohman, \& Woltz, 1984; Lohman, 1979, 1988). As an example, Barratt (1953) showed that the GZSOT involves various strategies for a solution other than VSPT, like mental rotation of the array, and stretching a mental line between the object and the target. 
Many studies that used psychometric tests of VSPT found a strong correlation between VSPT and mental rotation (Borich \& Bauman, 1972; Carroll, 1993; Goldberg \& Meredith, 1975; Price \& Eliot, 1975; Roff, 1952). Both VSPT and mental rotation rely on many common processes, such as the ability to encode spatial images and the ability to maintain these representations in memory (Hegarty \& Waller, 2004; Kosslyn, 1994). However, they also involve different cognitive processes; mental rotation relates to the ability to inspect changes in an object (or an array) when the observer remains steady and the object changes shape or position (Shephard \& Metzler, 1971). In contrast, VSPT relates to inspecting changes of an object's view (or an array) when the observer changes position and the object remains steady. In other words, mental rotation and VSPT are differentiated from each other in their spatial frame of reference. A frame of reference is a coordinate system that defines the location of an object in space. In mental rotation, the frame of reference is object based, whereas in VSPT, the frame of reference is egocentric (Zacks \& Michelon, 2005). This fundamental distinction is manifested in different patterns of behaviors. For example, in VSPT, participants commonly make egocentric systematic errors (e.g., they confuse directions relative to the self, like right and left) (Bryant \& Tversky, 1999; Hintzman, O’Dell, \& Arndt, 1981; Kozhevnikov \& Hegarty, 2001). In mental rotation, participants mentally rotate the object in a continuous manner, thus revealing a linear increase in response time as a function of the angular disparity between the real and the imagined positions (Dalecki, Hoffmann, \& Bock, 2012; Shepard \& Cooper, 1982; Shepard \& Metzler, 1971).

Unlike the psychometric approach, the cognitive approach uses experimental designs to explore VSPT, considering the sensory, imagery, and representational processes that are involved in it (Eliot, 1987). Various methods have been used, such as: navigation in mazes (e.g., Brown, 1932), sketching maps of learned environments (e.g., Lynch, 1960), deciding whether objects are seen or not (e.g., Surtees, Apperly, \& Samson, 2013; Wraga, Shephard, Church, Inati, \& Kosslyn, 2005) and deciding objects' quantities from imagined perspectives (e.g., Furlanetto, Becchio, Samson, \& Apperly, 2016). The most common method is judgment of relative direction (i.e., JRD) (Shelton \& McNamara, 1997; Wolbers \& Hegarty, 2010).

\section{Judgement of relative direction tasks}

In JRD tasks, participants are asked to mentally adopt a point of view (i.e., perspective) in space and to make a judgment about the mental direction of an object in relation to their body. For example, right now you are sitting at your desk in front of your computer. Imagine you are standing at the door, looking at the window. Now, indicate the direction to the bookshelf. In this example, you are asked to mentalize a new perspective of the room (imagine standing at the door, heading toward the window), and to make a judgment regarding a target object, the bookshelf. JRD tasks usually use a response board that is designed like a circle, with one stable line in it that is set upright. Participants are asked to move (e.g., Hintzman et al., 1981) or draw (e.g., Kozhevnikov \& Hegarty, 2001) a second line in accordance to the direction of the target object (see Fig. 1), so an angle is created between two vectors: the first one represents the heading direction (i.e., the window) from the imagined position (i.e., the door), and the second represents the estimated direction from the imagined position to the target object (i.e., from the door to the bookshelf). JRD tasks use memorized and familiar environments, that is, cognitive maps (e.g., Mou, McNamara, Valiquette, \& Rump, 2004; Shelton \& McNamara, 1997), or visual maps that are available to the subjects during the entire task and so do not involve memory of the layout (e.g., Hintzman et al., 1981; Kozhevnikov \& Hegarty, 2001). The advantage of JRD tasks is that they allow a continuous measure of performance (i.e., the error size, which is the disparity between the real and response angle), unlike other tasks that only measure binary, correct or incorrect responses. This enables revealing the strategy that was implemented for solving the task (e.g., Kozhevnikov \& Hegarty, 2001).

Two distinct angles are created in JRD tasks. One is a result of perspective change - the disparity between the actual, sensorimotor perspective, and the imagined perspective. The second angle is a result of the estimated direction to the target from the imagined perspective; it is the angle created between the vector of the imagined location (i.e., stationary object) and the heading object, and the vector of the stationary object and the target object (see Fig. 1). The disparity between the participants' answers (i.e., angles) and the real direction to the target is termed the error size (i.e., accuracy measure). Studies showed that error sizes vary as a function of perspective changes because the larger the perspective changes, the larger the discrepancy between what we see and need to imagine (e.g., Bryant \& Tversky, 1999; Hintzman et al., 1981; Shelton \& McNamara, 1997). Moreover, the pattern of accuracy and response time (RT) as a function of the estimated direction to the target is suggestive of a body-centered coordinate system. For instance, when participants estimate the direction to a target object that is mentally located in front of them, they are more accurate and faster than to a target object that is to their right or left (Bryant \& Tversky, 1999; Hintzman et al., 1981).

Kozhevnikov and Hegarty (2001) developed and validated a psychometric test - the object perspective test for VSPT ability - using the JRD method that so far has been used in experimental designs. Their aim was to demonstrate a dissociation between mental rotation and VSPT by creating a test that measures VSPT in a pure manner, without involvement of 

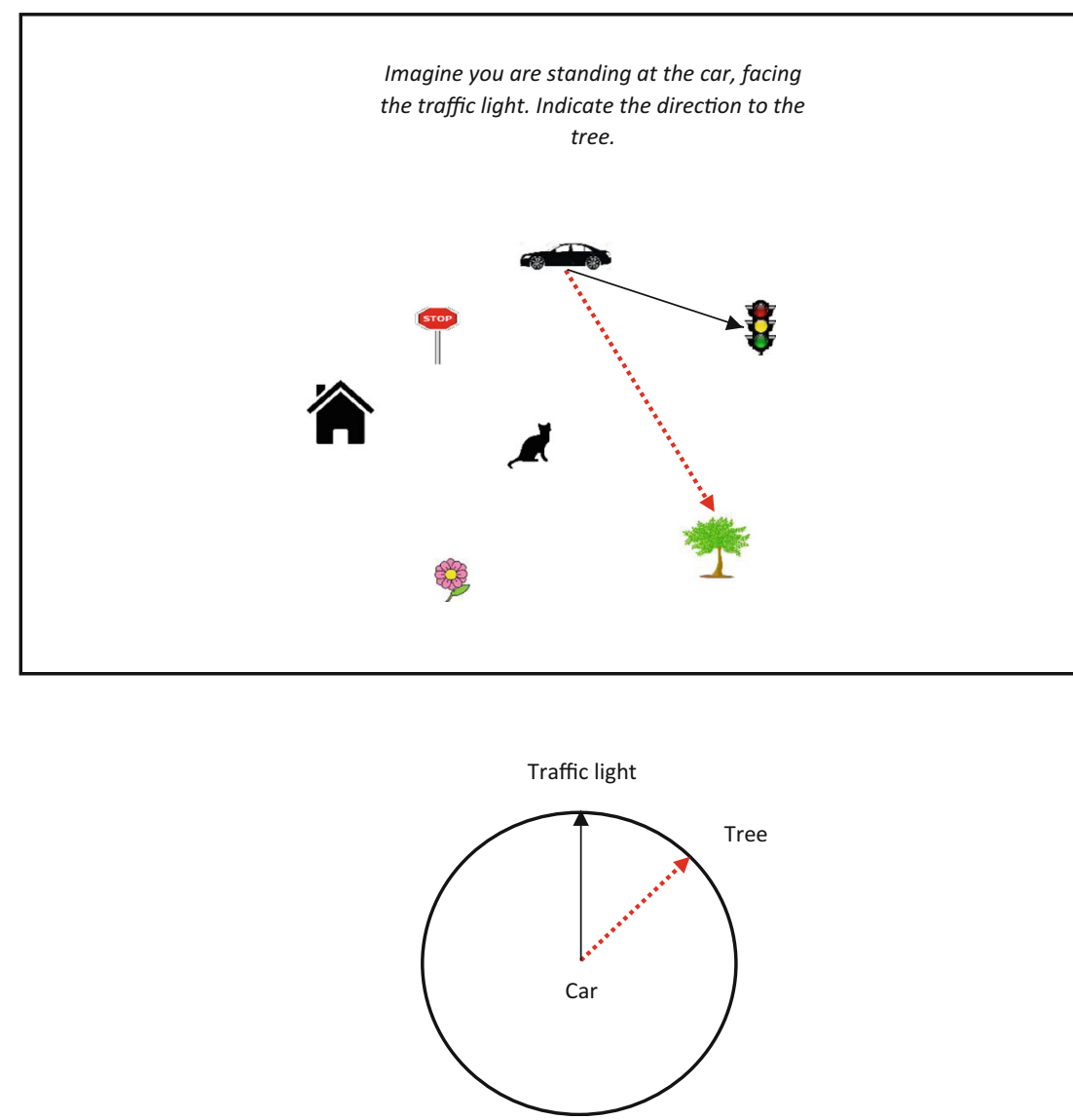

Fig. 1 Illustration of a trial in the object perspective test (Kozhevnikov \& Hegarty, 2001). Participants were asked to imagine being at the position of a stationary object in the display (the car) while facing another object (traffic light) - defining the imagined perspective within the array - and were asked to indicate the direction to a target object (tree) by stretching a line on a response board (i.e., a circle). The solid arrow illustrates the fixed line, which represents the imagined perspective in the objects layout, whereas the dashed arrow illustrates the answer (i.e., the direction to the target object). Note that in the given example, the perspective change is $110^{\circ}$ and the direction to the target object is $47^{\circ}$ other strategies (see also Hegarty \& Waller, 2004). In the object perspective test (see Fig. 1 for illustration), JRD is implemented with a visual map of seven objects that determine the imagined perspective and the target object, and participants are asked to enter their response (the direction to the target object) by drawing a line in a circle that already has a fixed line in it that is set upwards. The fixed line represents the perspective, so that participants are required to imagine they are standing in the center of the circle and the line is set to the heading object. Then, participants are asked to draw another line from the center to the circle's edge that represents the target object's direction (see Fig. 1).

Kozhevnikov and Hegarty (2001) argued that the analyses previously conducted for validating VSPT in JRD tasks analyzing error sizes as a function of perspective changes, and analyzing error sizes as function of body direction - are not sufficient to distinguish between VSPT and other strategies. The former analysis is not sufficient since the perspective change also determines the disparity between what one sees and imagines, so the errors would be larger in the case where a mental rotation strategy is implemented. The latter analysis is inadequate since front and back directions form almost a straight line, and as such, implementing an analytic strategy (i.e., estimating the angle without mentalizing rotation or taking an imagined perspective) would also yield smaller errors compared to right and left directions. Kozhevnikov and Hegarty's novelty was that they carried out an analysis of reflection errors. In VSPT, unlike in mental rotation, participants assume to perform mistakes that are systematic and correspond to the body's coordinate system. Thus, Kozhevnikov and Hegarty showed that participants tended, for instance, to confuse between right and left or front and back. If the test was solved using a mental rotation strategy only, the errors should not have corresponded to an egocentric frame of reference. Nevertheless, analyzing the participants' error patterns and asking the participants to report the strategies they used in the test, Kozhevnikov and Hegarty concluded that participants did not use VSPT for items involving perspective changes smaller than $90^{\circ}$ (see also Barratt, 1953; Hegarty \& Waller, 2004). Participants reported they solved those trials by mentally rotating the angle created by the objects in the trial to align with the circle's lines, or used an analytic strategy. 


\section{Perspective switching}

In the object perspective test, like in most of the JRD tasks that were studied so far (e.g., Hintzman et al., 1981; Shelton \& McNamara, 1997), the response board's (i.e., the circle) lines always appear in the same orientation, which is aligned with the observer's sensorimotor perspective. Thus, participants are required to perform VSPT for evaluating the directions of the target objects, and then to move back to the sensorimotor perspective in the response phase (Avraamides \& Kelly, 2008).

Studies found that performance in JRD tasks was faster and more accurate when participants responded verbally than when they pointed physically to the target's direction (e.g., Avraamides \& Ioannidou, 2005; de Vega \& Rodrigo, 2001). Therefore, Avraamides and Ioannidou (2005) concluded that when a decision about a direction is mentalized, participants are required to ignore their physical orientation. Thus, "going back" to a sensorimotor perspective makes the task harder. The current study aimed to investigate this process of "moving between perspectives" in a JRD task, and, henceforth, we will refer to this process as perspective switching.

Exploring the perspective switching component is interesting, first because it may help in understanding the processes involved in JRD tasks, and possibly it can be adjusted for measuring VSPT more "purely." Additionally, switching between sensorimotor and mental perspectives is a fundamental component in the complex process of VSPT (May, 2004). When taking a novel perspective, we constantly switch between what we see (i.e., sensorimotor perspective) and what we mentalize (i.e., imagined perspective). Thus, examining the process of perspective switching may teach us more about VSPT ability.

Accordingly, the current study used a JRD task - the dots perspective task (DPT) - to explore perspective switching. We manipulated the congruency between the perspectives taken in two phases within a trial, as is detailed later. A typical trial in the DPT is presented in Fig. 2.

\section{The dots perspective task (DPT)}

The DPT is a computerized task with a simple design and a response method that preserves the visual-spatial nature of the task, without the need for verbal processing for defining the imagined perspective and the target object (see Fig. 2 for an example of a trial).

In the DPT, participants are presented with an array of dots that change their colors (but not their location) from trial to trial. They are asked to mentally locate themselves in a position (represented by a smiley face), to mentally face towards a heading dot (the yellow dot in Fig. 2), and to estimate the direction to a target dot (i.e., the red dot in Fig. 2).
In Kozhevnikov and Hegarty's (2001) test, only total RT was recorded and only the accuracy measure was analyzed. In contrast, in the DPT, two RT measures are recorded since each represents a distinct phase of the trial: (1) imagining the position, heading, and estimated direction to the target (mental transformation phase), and (2) entering the estimated direction in the circle (response phase). Measuring both RTs separately enables exploring whether participants implemented a mental rotation strategy in the mental transformation phase; if participants use mental rotation, the correlation between the angular disparity (between real and imagined perspectives) and response RT (measured from the onset of the circle display) would be linear. Mental rotation could be implemented after the appearance of the response circle, since participants rotate the smiley and the heading dot to align the circle's fixed line, and then set the dynamic line to align to the direction of the target dot (see Hintzman et al., 1981). If there is no correlation between angular disparity and response RT, mental rotation was probably not used. In addition, the RT of the mental transformation phase is a marker of the effort participants invested in imagining the new perspective and mentally estimating the direction to the target, before the response circle appeared. A short mental transformation RT could indicate that no effort was invested in imagination of the new perspective or mental estimation of the target's direction.

The stimuli, instructions, perspective changes, and targets' directions are described in more detail in the method section.

We were interested in examining both switching from a sensorimotor perspective to an imagined perspective and switching from an imagined perspective to a sensorimotor perspective within a trial in the DPT. To this end, we manipulated the congruency between perspectives (sensorimotor or imagined) in the two phases of the task, that is, the mental transformation phase and the response phase. The perspectives in both phases were manipulated using the smiley's (the stationary object's) position and the setting of the circle's fixed line that could be congruent with the smiley's position or incongruent with it, as will be explained further.

In each trial, the smiley is positioned above the layout or below it, to create two conditions: (1) When the smiley is below the dots' layout, it aligns with the observer's view of the screen, and as such, the representation of space is egocentric (i.e., representation of space "as we see it"). For instance, the yellow dot in the trial demonstrated in Fig. 2 is to the right of both the smiley and the participant's body. Moreover, when the smiley is below the layout, all the perspective changes are smaller than $90^{\circ}$, thus those trials do not require implementation of VSPT (Hegarty \& Waller, 2004; Kozhevnikov \& Hegarty, 2001). (2) When the smiley is above the layout, it creates perspective changes that are larger than $90^{\circ}$, thus requiring VSPT. Henceforth, we refer to the smiley's positions as " $<90 "$ when the smiley is below the layout and " $>90 "$ when the smiley is above the layout. We examine if participants 


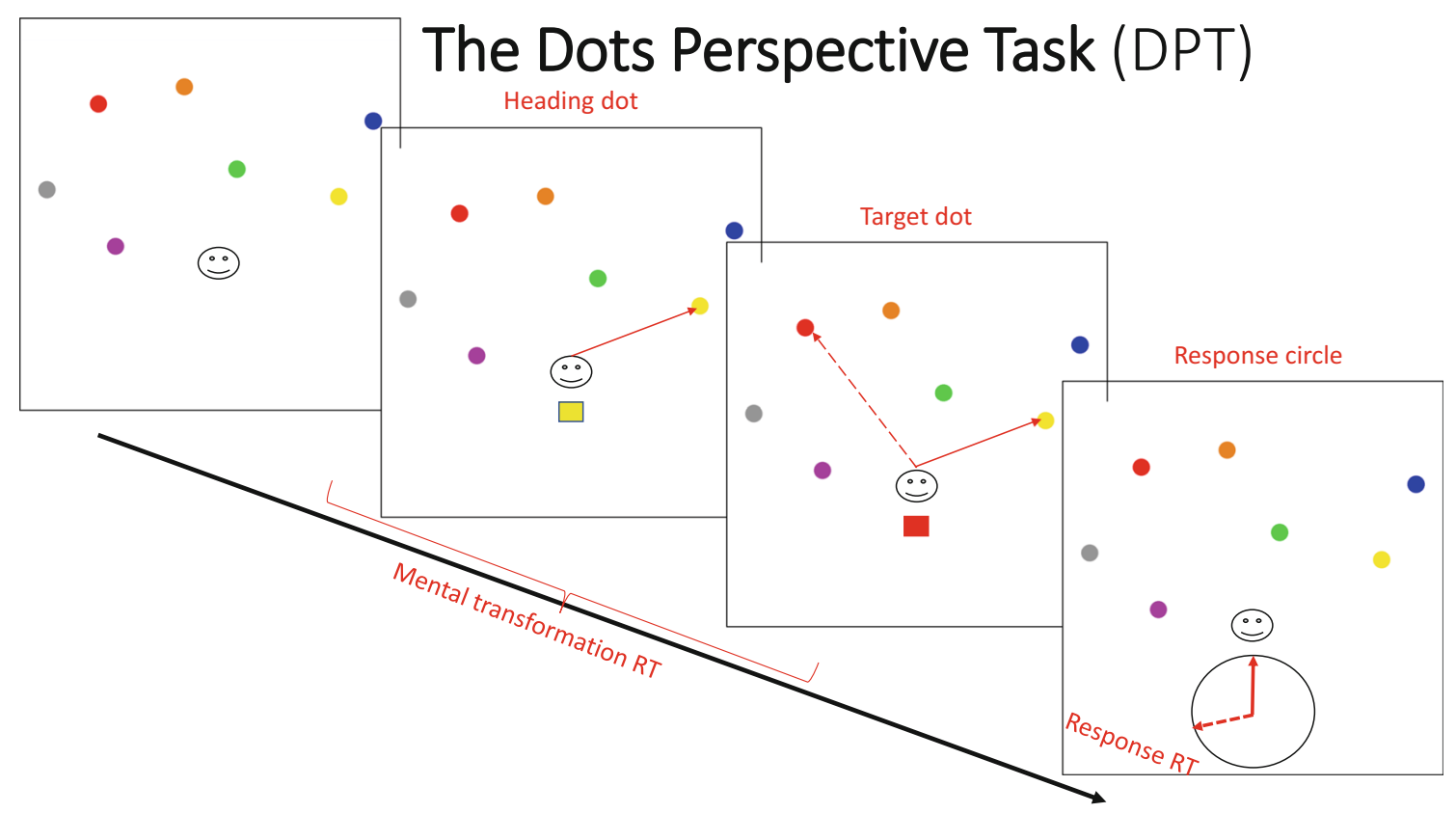

Fig. 2 The dots perspective task - a schematic description of a trial. There are two lines in the circle: a fixed line and a dynamic line that can be moved by pressing the arrow bars on a computer keyboard. Each screen is presented until the participant presses the space bar. Two measures of response time (RT) are recorded: (1) the time it takes participants to mentalize their perspective and indicate the direction to the target dot (i.e., "mental transformation RT", starting from the beginning of the trial

employ mental rotation in the conditions that are termed as $<90$, as Kozhevnikov and Hegarty (2001) suggested, by analyzing whether the time it takes to enter the response (i.e., "response RT") correlates with the angular disparity between the imagined and the sensorimotor perspectives (i.e., the perspective change) (Hintzman et al., 1981; Shepard \& Metzler, 1971).

The circle's lines could appear upright (be set to 12 o'clock) or face downwards (be set to 6 o'clock). When the line is upright, the perspective is compatible with the participant's sensorimotor perspective. When the line faces downwards, in contrast, the participants are required to execute a mental transformation because the perspective in the circle is incompatible with their sensorimotor perspective.

Manipulating the perspectives in the layout and in the circle creates four distinct conditions (a schematic description of the various conditions in the study is presented in Fig. 3): (1) The smiley is below the array and the circle's fixed line is facing upwards - participants are not expected to activate a VSPT process, since the perspective changes in the layout are smaller than $90^{\circ}$. In that case, the perspectives in the dots' layout and in the circle are congruent to each other (i.e., sensorimotor), thus there is no switch between sensorimotor and imagined perspectives. We consider this condition as a baseline condition since neither a VSPT nor a switch between perspectives is required. (2) The smiley is below the layout and the circle's fixed line is facing downwards - participants are not until the participant presses the space bar after screen \#3); and (2) the time it takes participants to tap their responses in the circle once it appears (i.e., "response RT", starting at the appearance of screen \#4 until the end of the trial when the participant presses the space bar). Note that the lines in the figure that represent the heading direction and the target direction are for illustration only, and do not appear in the task

required to perform VSPT in the dots' layout phase but are required to switch from a sensorimotor perspective to an imagined perspective in the response phase. This condition enables examining switching from a sensorimotor perspective to a mental perspective and comparing it to switching from a mental perspective to a sensorimotor perspective (see condition \#4). (3) The smiley is above the layout and the circle's fixed line is facing down - both the perspectives in the dots' layout phase and in the circle are imagined and thus congruent. We assume participants "stay" in the imagined perspective when they are entering their answers in the circle. We are mostly interested in comparing between conditions 3 and 4 . (4) The smiley is above the layout and the circle's fixed line is facing upwards - this condition represents a typical trial in the object-perspective test where participants are required to perform VSPT in the layout and to move to a sensorimotor perspective in the response phase, so the perspectives are incongruent. We are interested in examining the cost of switching from an imagined perspective to a sensorimotor perspective, in relation to staying in the imagined perspective, and in relation to switching from a sensorimotor perspective to an imagined perspective.

If switching between perspectives (i.e., incongruent trials) does not require an extra cost in performance then one could expect no congruency effect. We postulate that switching between the sensorimotor and mental perspectives in the layout and the circle is relevant and should be taken into 


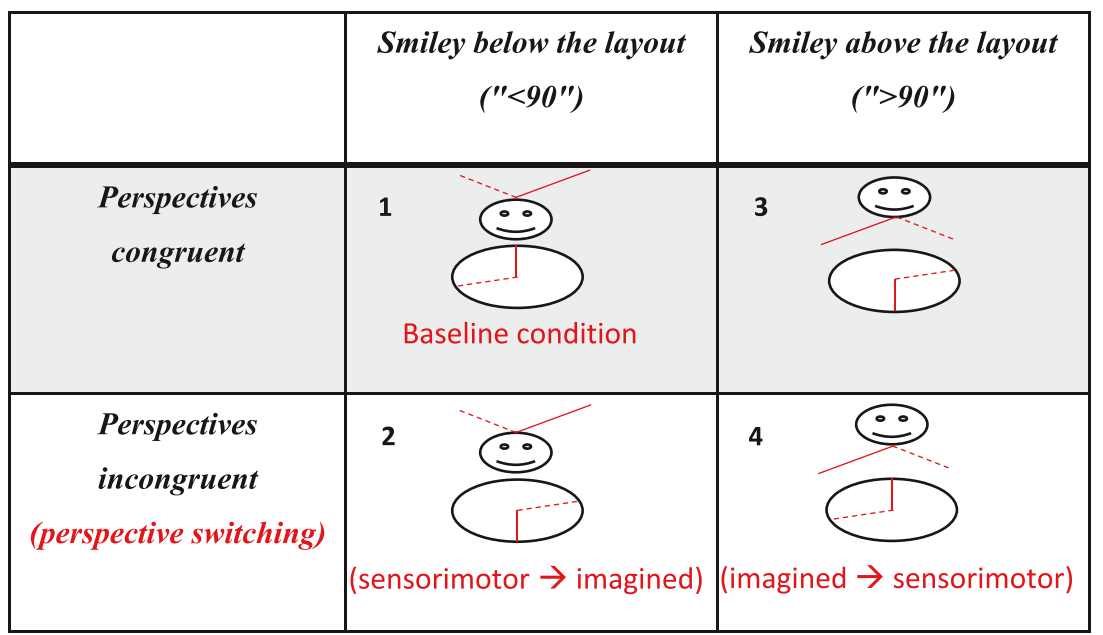

Fig. 3 A schematic description of the four conditions in the dots' perspective task. Congruency is defined by the relation between the perspectives (sensorimotor/imagined) in two phases of the task, that is, before and after the display of the response circle (see text for details in each condition)

consideration specifically in the current task, and in JRD tasks in general. Analyzing accuracy and RT measures will allow us to explore the cost of perspective switching and to clarify the mental transformation processes that are involved in the DPT. Mental rotation is explored using response RT analysis as a function of perspective changes, and VSPT is explored using mental transformation RT analysis, and using Kozhevnikov and Hegarty's (2001) suggested analysis of reflection errors. In summary, the current study presents a computerized JRD task that enables exploration of VSPT, mental rotation and perspective switching.

Finally, we used two groups of participants with different dot configurations (one configuration was a mirror image of the other, on the $\mathrm{x}$ - and the $\mathrm{y}$-axis) to examine whether the task results were replicated across configurations.

\section{Experiment 1}

\section{The study hypotheses}

First, it was postulated that the group variable (i.e., dot arrangement) would not interact with other variables, so the results would be replicated with both configurations of dots. Second, we expected to find more reflection-errors (mistaking right and left) in the $>90$ condition than in the $<90$ condition, since perspective changes larger than $90^{\circ}$ involve a VSPT strategy (e.g., Kozhevnikov \& Hegarty, 2001), and as Kozhevnikov and Hegarty showed, VSPT leads to systematic errors that correspond to the body coordinate schema. Third, we expected that the time to indicate the answers in the circle (i.e., response RT) would not be correlated with the perspective changes in the $>90$ condition. A non-significant correlation would not support the possibility that participants implemented a mental rotation in that condition (when the smiley is above the layout). In contrast, we expected the response RTs to be correlated with the perspective changes in the $<90$ condition because we assumed participants would employ mental rotation in order to align the smiley and the heading dot in the layout with the circle's fixed line (Hintzman et al., 1981). Finally, we expected to find larger errors and longer response RT in the incongruent trials, both in the $<90$ and the $>90$ conditions, because participants are required to switch between a sensorimotor and an imagined perspective, and between an imagined and a sensorimotor perspective, respectively.

\section{Method}

Participants As far as we know, this is the first study that explores perspective switching while examining the congruency between the perspectives in the dots' layout and in the response circle. Moreover, most of the studies that used a JRD task did not report effect sizes (e.g., Hintzman et al., 1981; Kozhevnikov \& Hegarty, 2001). We expected small-medium effect sizes in line with May's (2004) findings in a study that used a JRD task and explored switching between sensorimotor and mental perspectives (see Experiment 1 in May, 2004). A power analysis using $\mathrm{G}^{*}$ Power 3.1 (Faul, Erdfelder, Lang, $\&$ Buchner, 2007) indicated that the needed sample size for examining the effect of perspective switching at a power $>95 \%$ to test an effect size of 0.2 with a Type 1 error $(\alpha<$ 0.05 ) is 56 participants. We enlarged our sample to 69 participants to be prepared for dropouts and exclusions. Note that in Experiment 2 we used 56 participants for replicating the results with the recommended sample size, and post hoc Bayesian analyses were conducted and reported.

The participants included 69 undergraduate students (mean age $=23.66$ years, $S D=1.99$ ) recruited from the subject pool at Ben-Gurion University of the Negev, Israel. The participants were compensated with payment of 35 shekels 
(approximately US\$10) for their participation. All participants reported normal or corrected-to-normal vision and had no attention deficit disorder or learning disability. All participants gave written informed consent prior to the commencement of the experiment, which was approved by the behavioral ethics committee of the university.

Materials In each trial in the DPT, a configuration of seven dots in different colors (red, brown, yellow, green, purple, gray, and blue) appeared on the upper part of the screen, with a smiley (-) positioned randomly above or below the dots. The location of the dots was fixed across the trials, but their colors changed randomly over the trials. Participants were asked to imagine themselves located in the position of the smiley and to press the space bar on the keyboard when they succeeded imagining it (Fig. 2, screen 1). Next, a colored square appeared beneath the dots' layout, indicating the color of the heading dot. Participants were asked to press the space bar when they succeed in imagining their mental perspective (Fig. 2, screen 2 ). Then, the colored square disappeared for $150 \mathrm{~ms}$ in order to prevent masking of the colored squares and subsequently another colored square appeared beneath the layout, indicating the color of the target dot. Participants were asked to estimate the target dot's direction from their imagined perspective. Again, after completing this step mentally, the participants were asked to press the space bar (Fig. 2, screen 3). Finally, a circle with scale marks of degrees $\left(0-360^{\circ}\right)$ appeared at the bottom of the screen, containing two lines either pointing up or down. One line was fixed, and the other was dynamic and was controlled by the right and left arrows on the keyboard. Here, participants were guided to imagine themselves standing at the center of the circle, using the fixed-line heading to the edge of the circle to represent their mental perspective. They were asked to indicate the direction to the target dot by moving the dynamic line to represent the target's direction. After setting the angle on the circle, participants were asked to press the space bar to proceed to the next trial (Fig. 2, screen 4). Due to the manipulation of the perspectives in both the dots' layout phase and the response phase, it was very hard not to confuse between right and left when indicating the target's direction. Thus, in order to facilitate performance, participants were instructed to follow the direction to the target in accordance with the right and left side of their body, by imagining the dynamic line was their right or left hand.

The researchers verified that participants understood the instructions and were able to discriminate between right and left. Participants were instructed not to rotate themselves in their seats but to use their imagination during the task. Moreover, participants were asked to verbalize their actions in the practice trials, to make sure they followed the instructions.

Procedure Data collection and stimulus presentation were controlled by a DELL OptiPlex 760 vPro computer with an
Intel core 2 duo processor E8400 $3 \mathrm{GHz}$. Stimuli were displayed on a 23-in. LED monitor (Dell E2314Hf) at a resolution of $1,920 \times 1,080$ pixels. A keyboard was placed on a table between the participant and the monitor. Participants were tested individually and sat approximately 40 in. from the computer screen. Stimulus presentation and data acquisition were controlled by Psychtoolbox software (version 3.0.11) (Brainard, 1997; Pelli, 1997) in MATLAB (MathWorks version 8.4.0.150421 (R2014b)). The dots' diameter was $1.8 \mathrm{~cm}$ (i.e., visual angle of $2.58 \mathrm{~cm}$ ), the smiley's size was $1.4 \mathrm{~cm}$ (i.e., visual angle of $2 \mathrm{~cm}$ ) and the circle's diameter was $8.8 \mathrm{~cm}$ (i.e., visual angle $12.55 \mathrm{~cm}$ ). See Fig. 4 for stimulus proportion and distances.

Prior to the task, participants read seven slides of instructions and then completed eight practice trials, three of which were accompanied by a researcher's explanations and demonstrations. Then they carried out the task, which included 128 trials, divided into two blocks of 64 trials each, with a break in the middle. Participants were tested individually on the task, which lasted approximately 45 min. The participants were allocated arbitrarily to one of two groups (group 1: $\mathrm{N}=39$ and group 2: $\mathrm{N}=30$ ). Both groups completed the same experiment, but with different dot configurations. The configurations were a mirror image of each other (flipped on the $\mathrm{x}$ - and the $\mathrm{y}$-axis), so that the same targets' directions that were applied in the $>90$ condition in one group were applied in the second group in the $<90$ condition.

Design There were two within-subject independent variables (i.e., perspective and congruency) and one between-subject variable (i.e., group; for two dots' configuration arrangements) in the design. The perspective variable was either imagined (i.e., the smiley positioned above the dots layout ">90") or sensorimotor (i.e., the smiley positioned below the dots' layout - "<90"). The congruency variable was either congruent (i.e., the perspectives in the dots' layout phase and in the response phase were similar — imagined/sensorimotor) or incongruent (i.e., the perspectives in the dots' layout phase and in the response phase were not similar). See a schematic description of the four task conditions in Fig. 3.

Two variables were controlled in the DPT - the perspective changes and the target directions.

Perspective changes The perspective changes are the disparities between the sensorimotor perspective that aligns with the view of the screen, and the vector created from the smiley to the heading dot. In the first group, the perspective changes were: $17^{\circ}, 41^{\circ}, 50^{\circ}, 62^{\circ}, 68^{\circ}$, and $81^{\circ}$ for the $<90$ condition, and $110^{\circ}, 115^{\circ}, 130^{\circ}, 140^{\circ}$, and $156^{\circ}$ for the $>90$ condition. In the second group, the perspective changes were: $24^{\circ}, 41^{\circ}, 50^{\circ}$, $65^{\circ}$, and $68^{\circ}$ for the $<90$ condition, and $99^{\circ}, 111^{\circ}, 118^{\circ}, 130^{\circ}$, $140^{\circ}$, and $163^{\circ}$ for the $>90$ condition. 


\section{•}
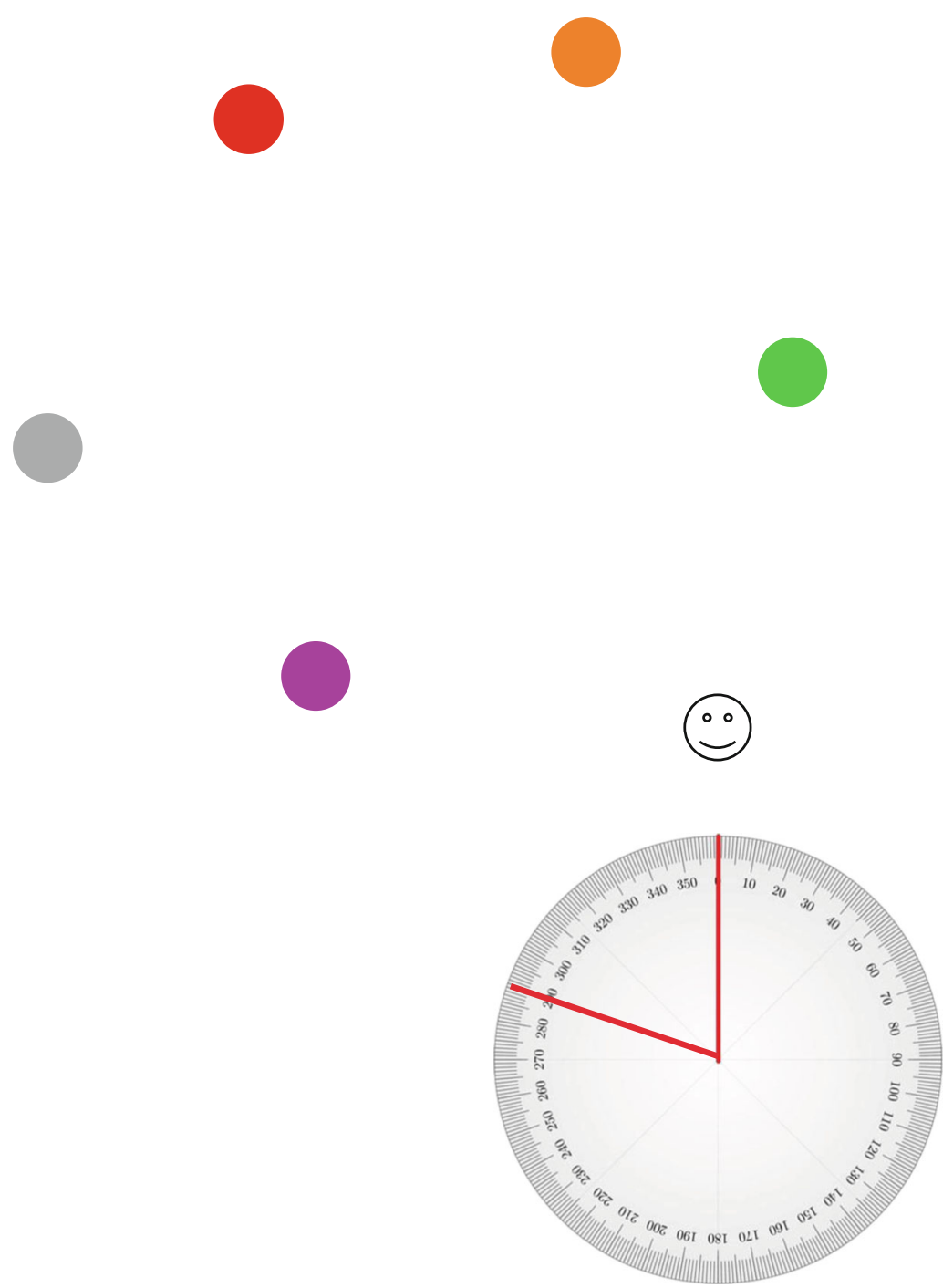

Fig. 4 The view of the screen during the experiment in one of the trials. Note that the dot positions were fixed during the experiment, but their colors were changed arbitrarily. The fixed line in the circle was set upwards/downwards and the second line was dynamic and appeared

Target directions The target directions are the angles created between the vector from the smiley to the heading dot and the vector from the smiley to the target dot. The angles for the $>90$ condition in the first group were: $26^{\circ}, 60^{\circ}, 107^{\circ}, 133^{\circ}, 227^{\circ}$, $253^{\circ}, 300^{\circ}$, and $334^{\circ}$. For the second group, these angles were created for the $<90$ condition. The angles for the $<90$ condition in the first group were: $45^{\circ}, 74^{\circ}, 117^{\circ}, 146^{\circ}, 214^{\circ}, 243^{\circ}$, $286^{\circ}$, and $315^{\circ}$. These angles were created for the $>90$ condition in the second group.

The perspective changes determined the rotation discrepancy in trials where participants employed a mental rotation strategy for a solution. Hintzman et al. (1981) showed that in a JRD task, participants mentally rotated the relevant information in the layout (i.e., the smiley defining the imagined position, the heading dot and the target dot) to be aligned with the lines in the response board (i.e., the circle). The rotation behind the fixed line at the beginning of the trial. Moreover, the circle appeared on the screen after participants pressed the space bar three times (see Fig. 2)

discrepancy was calculated for each condition in the task, in accordance with the perspective change and the circle's lines position, assuming that participants were mentally rotating the stimuli in the shortest direction (Hintzman et al., 1981).

Scoring There were four dependent measures: (1) Absolute error size - the absolute discrepancy between the angle the participants entered in the circle and the actual direction to the target. This measure indicates participant accuracy and was previously attributed to VSPT ability (Hegarty \& Waller, 2004; Kozhevnikov \& Hegarty, 2001). (2) Mental transformation RT - the time it took participants to imagine the different stages in the dots' layout, that is, the RT from the beginning of the trial until the end of the third screen (see Fig. 2). (3) Response RT - the time it took participants to initiate moving the dynamic line in the circle and to set the angle in it. (4) 
Table 1 Descriptive statistics (means) of dependent variables in Experiment 1

\begin{tabular}{lcccr}
\hline Condition & Reflection error rate & Absolute error size $\left(^{\circ}\right)$ & Mental transformation RT (s) & Response RT (s) \\
\hline$<90$ & $0.04(0.03-0.05)$ & $17.38(14.15-20.62)$ & $5.8(5.6-6.01)$ & $7.2(6.87-7.54)$ \\
$\begin{array}{l}\text { Congruent (baseline) } \\
\text { Incongruent }\end{array}$ & & & $9.05(8.83-9.27)$ \\
$>90$ & $0.08(0.07-0.09)$ & $24.39(21.71-27.08)$ & $7.1(6.89-7.31)$ & $9.06(8.73-9.39)$ \\
Congruent & & $33.73(28.1-39.36)$ & $8.69(8.32-9.07)$ \\
Incongruent & & & \\
\hline
\end{tabular}

Note. Confidence intervals (Loftus \& Masson, 1994) are given in parentheses

Reflection errors - the proportion of trials in which participants confused directions in their responses. Reflection errors indicate egocentric errors, which are attributed to execution of a VSPT strategy (Kozhevnikov \& Hegarty, 2001). We analyzed reflection errors to explore the strategies that were employed in the $>90$ and the $<90$ conditions. If, for example, a participant estimated the target direction to be approximately $315^{\circ}$ when it was actually $45^{\circ}$, he/she probably confused directions between right and left. Kozhevnikov and Hegarty classified errors as reflection errors if they were within $\pm 20^{\circ}$ of a response that was a reflection of the correct response through the horizontal (right and left), vertical (front and back), or both (right-front, right-back, etc.) axes. For our purpose, we only used the analysis of the horizontal axis, namely, we analyzed only right-left direction mistakes. We hypothesized that participants would commit more reflection errors in the $>90$ condition compared to the $<90$ condition because of the different strategies implemented in these two conditions (Kozhevnikov \& Hegarty, 2001). Note that we referred to a reflection error only in trials with direction mistakes (between right and left) and with a deviation of $\pm 20^{\circ}$ from the correct answer.

\section{Results}

Reflection errors exceeding $50 \%$ of the response rate in the baseline condition ( $<90$, congruent trials, see Fig. 3) might indicate participants did not do the task or were not able to distinguish between left and right. Three participants presenting this proportion of reflection errors (two from group 2 and one from group 1) were excluded from further analyses. Moreover, in both RT measures, trials exceeding \pm 2.5 standard deviations from the mean (that was computed for each participant in each condition) were excluded from the RT analysis. This procedure resulted in exclusion of $0.04 \%$ of the trials. Descriptive statistics are given in Table 1. Analyses were conducted using STATISTICA software, version 12 (StatSoft. Inc, 2014). Bayesian analyses were conducted using JASP software, version 0.9 (JASP Team, 2018).

Reflection errors as a function of perspectives We compared the rate of reflection errors in the $>90$ condition to the $<90$ condition. A mixed analysis of variance (ANOVA), with group $(1 / 2)$ as a between-subject variable and perspective as a within-subject variable, was conducted on the rate of reflection errors. The analysis revealed a significant main effect for perspective, $F(1,64)=7.56, p=.008, \eta_{p}^{2}=.11, B F(10)=$ 11.79 , indicating there were more reflection errors for the $>90$ condition than for the $<90$ condition. Neither the main effect of group nor the interaction of group and perspective were significant, $F(1,64)=1.27, p=.26, B F(10)=0.6$ and $F<$ $1, B F(10)=0.45$, respectively.

Absolute errors A three-way mixed ANOVA with perspective and congruency as within-subject variables and group as a between-subject variable was conducted on the absolute error sizes as a dependent variable. A significant main effect was found for perspective, $F(1,67)=10.88, p=.002, \eta_{p}^{2}=.15$, $B F(10)=25.45$, indicating bigger errors for the $>90$ condition than for the $<90$ condition. A significant effect was also found for congruency, $F(1,67)=9.79, p=.003, \eta_{p}^{2}=.13, B F(10)=12.74$, indicating incongruent trials had bigger errors than congruent trials. The interaction was not significant, $F<1, B F(10)=0.39$ (see Fig. 5).

Mental transformation RT We carried out a mixed ANOVA with group $(1 / 2)$ as a between-subject variable and perspective $(<90 /$ $>90$ ) as a within-subject variable on the mental transformation RT. Congruency was not considered in this analysis as it was irrelevant because the mental transformation RT measured the time to imagine the layout before the circle appeared. The analysis revealed a significant main effect for perspective, $F(1,67)=$ $34.86, p<.001, \eta_{p}^{2}=.35, B F(10)=121,164.4$, indicating mental transformation time was shorter for the $<90$ condition than for the $>90$ condition. There was no significant effect for group, $F=$ $1.62, B F(10)=0.65$, or for the interaction, $F<1, B F(10)=0.6$.

Response RT A mixed ANOVA with group (1/2) as a betweensubject variable and perspective $(<90 />90)$ and congruency (congruent/incongruent) as within-subject variables was performed on the response RT. We found significant main effects for perspective, $F(1,67)=17.43, p<.001, \eta_{p}^{2}=.21, B F(10)=$ 16.11 , indicating the response RT was longer for the $>90$ condition; and for congruency, $F(1,67)=12.12, p=.001, \eta_{p}^{2}=$ $.16, B F(10)=16.39$, indicating the response RT was longer for 


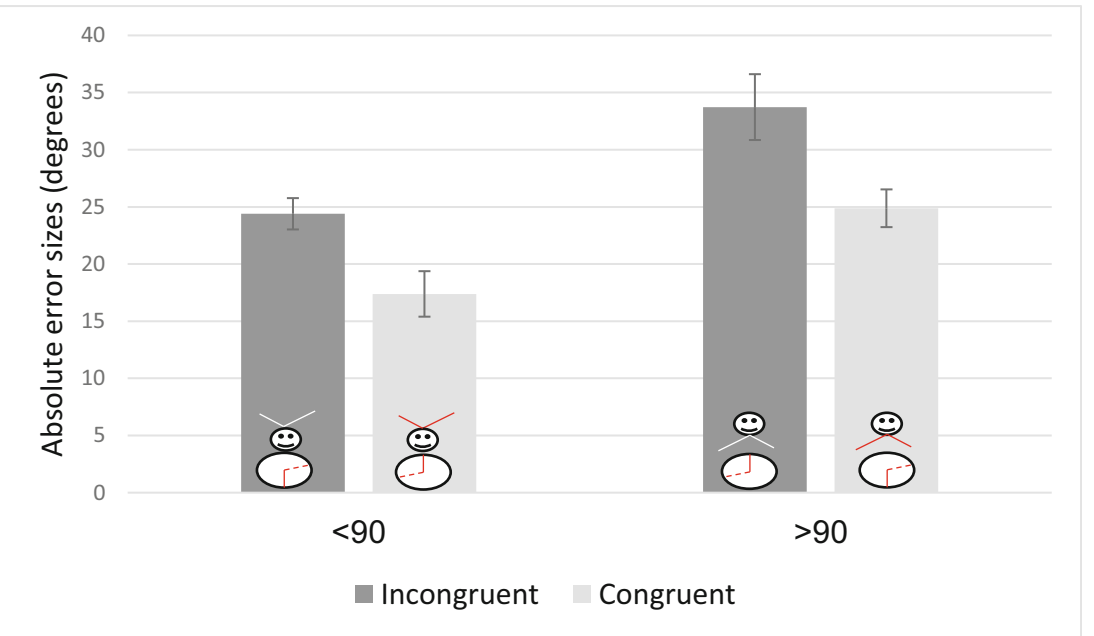

Fig. 5 Absolute error sizes as a function of perspective $(<90 />90)$ and congruency (incongruent/congruent). The cartoons are schematic representations of the conditions

incongruent trials. We also found a significant interaction for perspective $\times$ congruency, $F(1,67)=40.54, p<.001, \eta_{p}^{2}=$ $.39, B F(10)=13.98$. Further analysis revealed longer RTs for incongruent compared to congruent trials for the $<90$ condition, $F(1,67)=61.08, p<.001, \eta_{p}^{2}=.49$. No difference was found between incongruent and congruent trials in the $>90$ condition, $F<1$ (see Fig. 6).

Perspective changes (angular disparity) as a function of response RT A linear trend was examined using a Pearson bivariate correlation between the calculation for angular disparity and the response RT. The correlation was checked separately for the two perspectives ( $<90$ and $>90)$. In the $<90$ condition, the correlation between the angular disparity and the response RT was strong and significant, $r(18)=.795, p<.001$, whereas the correlation for the $>90$ condition was small and not significant, $r(20)=.036, p=.88$.

\section{Discussion}

The goal of the first experiment was to examine perspective switching in a JRD task involving perspective changes bigger than $90^{\circ}$ (i.e., $>90$ condition) that were previously attributed to a VSPT strategy (e.g., Kozhevnikov \& Hegarty, 2001), and perspective changes smaller than $90^{\circ}$ (i.e., $<90$ condition) that were attributed to implementation of mental rotation (e.g., Hintzman et al., 1981; Kozhevnikov \& Hegarty, 2001). We examined the strategies participants employed in the two conditions using analyses of accuracy and two distinct measures of RTs. We explored perspective switching when the (imagined/sensorimotor) perspective in the layout was incongruent to the perspective in the response phase (i.e., incongruent condition).

The results revealed: (1) more reflection errors, bigger absolute errors, and longer mental transformation RT for

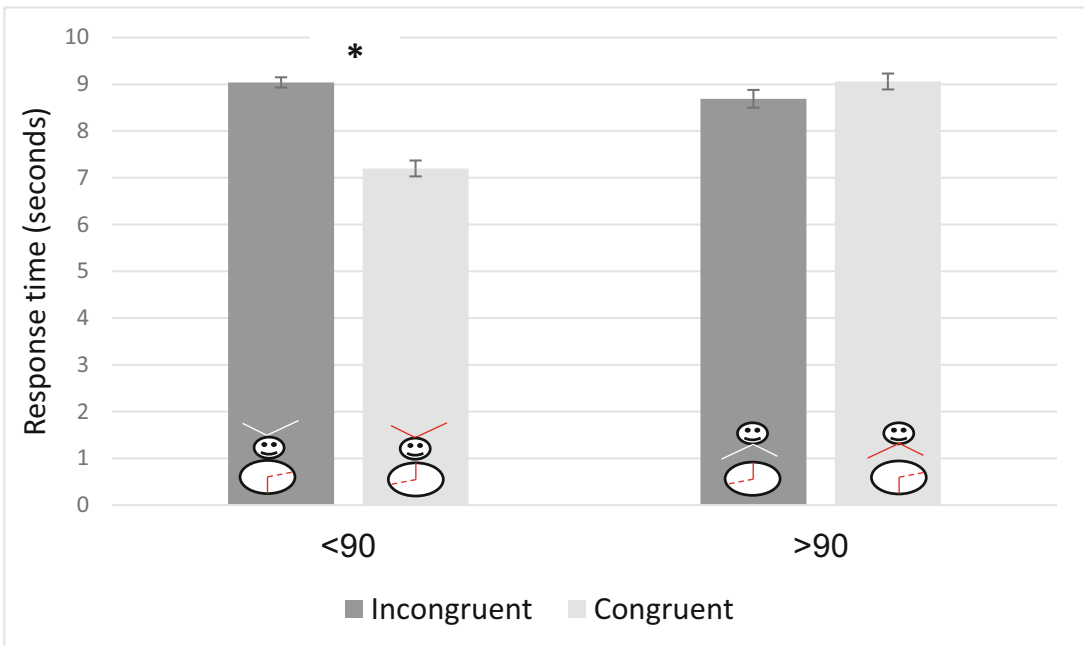

Fig. 6 The response RT as a function of perspective $(<90 />90)$ and congruency (incongruent/congruent). The asterisk $(*)$ represents a significant difference between columns 
the $>90$ condition compared to the $<90$ condition; (2) a significant correlation between the response RT and the angular disparity (i.e., perspective changes) in the $<90$ condition but not in the $>90$ condition; (3) bigger absolute errors for incongruent conditions; and (4) longer response RT for incongruent conditions in the $<90$ condition but not in the $>90$ condition.

We assumed that participants implemented a VSPT strategy in the trials where the smiley was above the layout, but not when the smiley was below the layout, since the perspective changes were smaller than $90^{\circ}$ in the latter (e.g., Barratt, 1953). More reflection errors in the $>90$ condition indicated that participants commonly used an egocentric frame of reference, namely, their mistakes corresponded to their body's axes (confusing right and left directions within a deviation of $\pm 20^{\circ}$ ) (Kozhevnikov \& Hegarty, 2001). Moreover, the longer mental transformation RT in the $>90$ condition, compared to the $<90$ condition, strengthened the notion that participants invested mental effort before the response circle appeared. Mental rotation would not require long RT before the circle appears on the screen, since the rotation of the layout requires information about the circle's fixed line position for mentally aligning the smiley and the heading dot with it (Hintzman et al., 1981). Interestingly, the response RT was also longer for the $>90$ condition compared to the $<90$ condition. It might indicate that the judgment phase is more effortful in the $>90$ condition trials compared to the $<90$ condition trials.

Kozhevnikov and Hegarty (2001) excluded trials with perspective changes smaller than $90^{\circ}$ from the object perspective test since their pattern of reflection errors did not correspond to the body coordinate system. In a subsequent experiment, the participants were asked to report the strategy they implemented in those trials, and one common strategy that was reported was mental rotation. Since the DPT is a computerized task, it enabled measuring the RTs at different stages of the task. In the $<90$ condition, the correlation between the response RT and the angular disparity (between the perspective in the layout and the circle's line position) was strong and significant. This correlation indicated that in this condition, participants might mentally rotate the smiley and the heading dot so that the vector (i.e., the linear direction that was mentally created between the smiley and the heading dot in each trial) would be aligned with the circle's fixed line, and then set the dynamic line in the orientation of the target dot (see Experiments 1 and 2 in Hintzman et al., 1981). In the $>90$ condition, the correlation between the angular disparity and the response RT was weak and non-significant, providing no indication for mental rotation.

Researchers who used visual maps in JRD tasks argued about mental rotation of the array without separating perspective changes smaller and larger than $90^{\circ}$ (e.g., Hintzman et al., 1981; Shelton \& McNamara, 1997) or they separated the perspective changes without recording separately the RTs in two phases of the task, due to the limitations of paper-and-pencil tests (e.g., Kozhevnikov \& Hegarty, 2001). Our RT analyses yielded findings that supported a dissociation between mental rotation and VSPT and suggest that mental rotation and VSPT are two distinct processes. Moreover, our results support Kozhevnikov and Hegarty's (2001) notion that participants implement a VSPT strategy in trials that involve perspective changes larger than $90^{\circ}$.

The results revealed a significant effect of congruency. The meaning of this effect is different for the $<90$ and $>90$ conditions because of the different strategies that were implemented in them. In the $<90$ condition, the significant difference in absolute error size and in RTs between the congruent and incongruent conditions could be explained by the difference in the angular disparity that was created in the incongruent compared to the congruent trials. The incongruent trials had larger disparity compared to the congruent trials $\left(99-163^{\circ}\right.$ compared to $17-81^{\circ}$, respectively), so the incongruent trials might have been harder and taken longer to rotate the layout. Thus, in the $<90$ condition, the congruency effect should not be attributed to switching between perspectives, but to mental rotation. In contrast, we suggest that incongruent trials in the $>90$ condition indicate a perspective switching component. In previous studies (e.g., Hintzman et al., 1981; Kozhevnikov \& Hegarty, 2001; Shelton \& McNamara, 1997) that used JRD tasks, the response board's (i.e., the circle) lines were set upright, assuming participants automatically "went back" to the sensorimotor perspective when indicating target direction. In our results, incongruent trials in the $>90$ condition led to bigger errors compared to the congruent trials. This finding might show that participants "stay" in an imagined perspective in their mind when approaching the response board, and thus succeed better when the perspective in the circle "stays" mental (i.e., congruent). This finding corresponds to Avraamides and Kelly's (2008) notion that participants must effortfully transform the computed response vector they hold in their mind, when they are asked to manually indicate their response in an incongruent, sensorimotor perspective. Interestingly, the incongruent trials did not consume longer response RT, in contrast to our expectations. This finding might indicate that the perspective switching is not an effortful process but is manifested in accuracy. Moreover, longer RTs in the incongruent trials compared to the congruent trials could indicate that participants employed a mental rotation strategy in the response board. However, the similar response RTs between the congruent and the incongruent trials in the $>90$ condition strengthened our notion that $>90$ condition trials do not involve mental rotation; thus, the $>90$ condition measures VSPT in a satisfying manner. The cost in error sizes should be taken into consideration since it is the suggested measure for VSPT ability in the object perspective test (Kozhevnikov \& Hegarty, 2001). 
Finally, the dot arrangement had no main effect and no interactions with other variables. This indicates that the pattern of results is not limited to one unique configuration or to the specific perspectives that were created by the dots' arrangements.

\section{Experiment 2}

JRD tasks usually involve memorizing a spatial layout with objects on a large scale (e.g., Hintzman et al., 1981; May, 2004; Shelton \& McNamara, 1997) or in a visual map on a small scale (e.g., Hintzman et al., 1981; Kozhevnikov \& Hegarty, 2001). The object perspective test (Kozhevnikov \& Hegarty, 2001) does not require memorizing the object layout and thus it avoids the involvement of memory in the VSPT test. Nevertheless, the layout in the object perspective test is fixed throughout the experiment, so subjects might memorize the configuration after a few trials. Retrieving a cognitive representation of the layout could yield deviations that are not a result of the studied process (VSPT), but of inaccuracies of the cognitive representation; thus, we wanted to avoid this as much as possible.

In Experiment 1, we used two configurations of dots with different groups of participants, with each group having a fixed configuration throughout the task (only the colors of the dots were changed). The current experiment used the same configurations with the same participants. One group of participants was presented with the two configurations in two separate blocks, and one group was presented with the two configurations in a mixed block. This arrangement enabled us to examine whether potential memorization of the layout of dots affected performance. We hypothesized that there would be no blocked versus mixed effect.

Moreover, in the current experiment, the participants used the computer mouse to indicate their responses on the circle, as opposed to the previous experiment where they used the right and left arrow keys on the computer keyboard. The use of the keys could have affected the RTs because larger angles (target directions) required more key presses. Therefore, we examined whether the correlations between the RT and the disparity of rotation would be replicated in the current experiment.

\section{Method}

The current experiment was the same as Experiment 1 except for the following changes. The subject pool consisted of 56 undergraduate students (mean age $=24.84$ years, $S D=1.88$ ) following the same selection criteria as in Experiment 1. The participants were allocated arbitrarily to two groups. One group performed the DPT with the two configurations sequentially, with one configuration per block (i.e., a total of two blocks, "the blocked group"), and the other group performed the task with the configurations changing randomly within each of the two blocks (i.e., "the mixed group"). In addition, the participants now used the computer mouse to indicate the angle in the circle.

\section{Results}

Examination of the direction mistake rate indicated that there were no participants with direction mistakes exceeding $50 \%$ in the baseline condition (i.e., $<90$, congruent condition), so no participants were excluded from the analyses. Only $0.045 \%$ of the trials were excluded for exceeding \pm 2.5 standard deviations from the RT means in the RT analyses. Descriptive statistics are given in Table 2.

Reflection errors as a function of perspectives We compared the rate of reflection errors in the $>90$ condition to the $<90$ condition. A mixed ANOVA with blocked/mixed as a between-subject variable and perspective (imagined/sensorimotor) as a within-subject variable was conducted on the rate of reflection errors. The analysis revealed a significant main effect for perspective, $F(1,54)=11.47, p=.001, \eta_{p}^{2}=.18$, $B F(10)=12.47$, indicating there were more reflection errors for the $>90$ condition than for the $<90$ condition. Neither the main effect of blocked/mixed, $F<1, B F(10)=0.39$, nor the interaction, $F<1, B F(10)=0.37$, of blocked/mixed and perspective were significant.

Absolute errors An analysis of absolute error sizes revealed a pattern similar to that in the previous experiment: A significant main effect was found for perspective, $F(1,54)=11.27$, $p=.001, \eta_{p}^{2}=.17$, although the Bayes factor $(B F(10))$ was 0.64 . There was also a significant main factor for congruency, $F(1,54)=7.05, p=.01, \eta_{p}^{2}=0.12, B F(10)=5.65$, indicating that the $>90$ incongruent conditions had bigger errors than the $<90$ congruent conditions. No significant effects were found for the mixed/blocked variable, $F<1, B F(10)=0.16$, or for the interactions, that is, perspective and congruency, $F(1,54)$ $=1.53, p=.22, B F(10)=0.57$; mixed $/$ blocked and perspective, $F<1, B F(10)=0.08$; and mixed/blocked and congruency, $F(1,54)=1.37, p=.25, B F(10)=0.2$ (see Fig. 7).

Mental transformation RT A mixed ANOVA with blocked/ mixed as a between-subject variable and perspective (imagined/ sensorimotor) as a within-subject variable on the mental transformation RT was performed. Congruency was not considered in this analysis as it was irrelevant for this RT since it measured the time before the circle appeared. The analysis revealed a significant main effect for perspective, $F(1,54)=21.77, p<.001, \eta_{p}^{2}=$ $.29, B F(10)=715.3$, indicating that the RT was shorter for the $<90$ condition than for the $>90$ condition. There was no significant effect for the mixed/blocked variable, $F<1$. 
Table 2 Descriptive statistics (means) of dependent variables in Experiment 2

\begin{tabular}{|c|c|c|c|c|}
\hline Condition & Reflection error rate & Absolute error size $\left(^{\circ}\right)$ & Mental transformation RT (s) & Response RT (s) \\
\hline $\begin{array}{l}<90 \\
\text { Congruent (baseline) }\end{array}$ & $.034(.027-.041)$ & $17.79(13-22.59)$ & $6.02(5.83-6.2)$ & $6.36(6-6.71)$ \\
\hline Incongruent & & $30.77(26.15-35.39)$ & & $8.05(7.7-8.39)$ \\
\hline $\begin{array}{l}>90 \\
\text { Congruent } \\
\text { Incongruent }\end{array}$ & $.057(.005-.064)$ & $\begin{array}{l}27.99(23.04-32.94) \\
32.74(27.59-37.9)\end{array}$ & $6.9(6.7-7.07)$ & $7.54(7.28-7.81)$ \\
\hline
\end{tabular}

Note. Confidence intervals (Loftus \& Masson, 1994) are given in parentheses

Response RT A mixed ANOVA with blocked/mixed as a between-subject variable and perspective (imagined/sensorimotor) and congruency (congruent/incongruent) as withinsubject variables was performed. We found no significant effect for perspective, $F(1,54)=2.34, p<.13$, namely, response RT was the same for the $<90$ and the $>90$ conditions. However, the Bayes Factor $(B F(10))$ for the perspective effect was 141.78. We found a significant main effect for congruency, $F(1,54)=12.31, p=.001, \eta_{p}^{2}=.19, B F(10)=11,160.46$, namely, response RT was longer for incongruent trials. We also found a significant perspective $\times$ congruency interaction, $F(1,54)=26.83, p<.001, \eta_{p}^{2}=.33, B F(10)=622.86$. There were no significant effects for the mixed/blocked variable, $F<$ $1, B F(10)=0.29$, or for the interactions between mixed/ blocked and the other variables. Further analysis revealed longer response RTs for incongruent compared to congruent trials in the $<90$ condition, $F(1,54)=33.29, p<.001, \eta_{p}^{2}=.38$. No difference was found between the congruent and incongruent trials in the $>90$ condition, $F<1$ (see Fig. 8).

Perspective changes (angular disparity) as a function of response RT A linear trend was examined using a Pearson bivariate correlation between the angular disparity and the response RT. The correlation was checked separately for the two perspective conditions $(<90$ and $>90)$. In the sensorimotor perspective ( $<90$ condition), the correlation between the disparity of rotation and the response RT was strong and significant, $r(18)=.728, p=.001$, whereas the correlation for the imagined perspective $(>90)$ was not, $r(18)=.11, p=.88$.

\section{Discussion}

The pattern of results in Experiment 2 was similar to that reported in Experiment 1, namely, we found significantly more reflection errors and longer mental transformation RT in the $>90$ condition (i.e., imagined perspective) compared to the $<90$ condition (i.e., sensorimotor perspective) - findings that strengthened the notion that a VSPT strategy was employed in the $>90$ condition. Moreover, we found a significant correlation between response RT and angular disparity in the $<90$ condition, but not in the $>90$ condition, as in the first experiment. This finding indicates use of a mental rotation strategy in the $<90$ trials, but not in the $>90$ trials. In addition, the results showed bigger absolute errors for the incongruent condition compared to the congruent condition and a significant interaction between perspective and congruency in the analysis of the response RT, as in the first experiment. Therefore, the results of the second experiment also support that switching between the imagined perspective in the layout

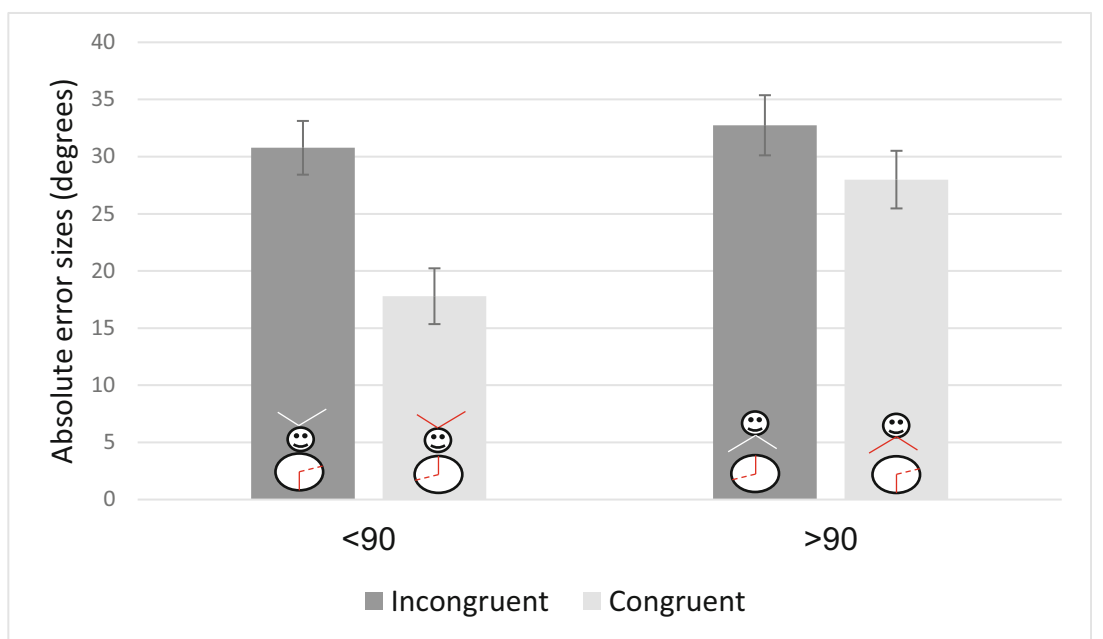

Fig. 7 Absolute error sizes (participants' responses) as a function of perspective $(<90 />90)$ and congruency (incongruent/congruent) 


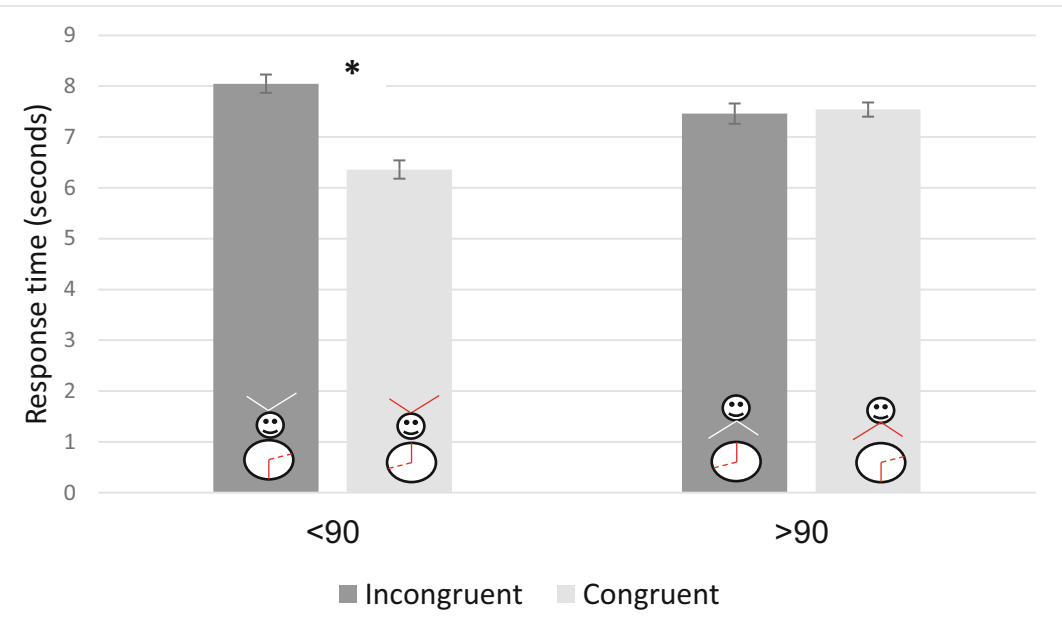

Fig. 8 The response RT as a function of perspectives $(<90 />90)$ and congruency (incongruent/congruent). The asterisk $(*)$ represents a significant difference between columns

to the sensorimotor perspective in the circle has a cost in accuracy, but not in RT. The blocked/mixed group had no significant effect or interaction with the other variables in any of the measures, thus we have no reason to suspect that the accuracy was affected by memorization of the layout. In summary, manipulating the dots' configurations in the layout between trials and between blocks did not affect the results. The response method (i.e., moving the computer mouse vs. pressing the arrows on the keyboard) did not change the results either.

\section{General discussion}

VSPT and mental rotation are two visual-spatial abilities that were found to be highly correlated and even un-dissociable (Carroll, 1993; Hegarty \& Waller, 2004). The current study suggests that the reported association between VSPT and mental rotation might be due to using impure tests for measuring VSPT (Kozhevnikov \& Hegarty, 2001).

JRD tasks were commonly in use for examining VSPT, with perspective changes that varied between $0^{\circ}$ and $180^{\circ}$ (e.g., Hintzman et al., 1981). Kozhevnikov and Hegarty (2001) showed that participants employ a VSPT strategy only in trials with perspective changes larger than $90^{\circ}$, and developed a JRD test for individual differences in VSPT. The accuracy (i.e., error sizes) in the test was analyzed and offered as a score. We introduced a computerized task that enables recording RTs in different phases of the task and uses simple stimuli in trials with perspective changes smaller or larger than $90^{\circ}$. Analyzing the RTs, in addition to accuracy, enabled further examination of the processes involved in the task. In addition, studies that use JRD tasks for examining (e.g., Hintzman et al., 1981) or measuring VSPT (Kozhevnikov \& Hegarty, 2001) commonly use a response method of a circle with a fixed line that is set upwards, and participants are required to draw or move a dynamic line to indicate the direction to a target. This response method requires switching from the imagined perspective to a perspective that aligns with the subject's sensorimotor perspective (Avraamides \& Kelly, 2008). The current study examined the congruency of the perspective taken in the dots' layout (i.e., mental transformation phase) and the perspective taken in the response circle (i.e., response phase). The perspectives could be congruent (not involve perspective switching) or incongruent (involve perspective switching).

The major finding of the current research is that in trials with a perspective change larger than $90^{\circ}$, when the perspective in the circle was incongruent to the imagined perspective, the error size was bigger compared to trials with a congruent perspective. We suggest that when performing VSPT, participants mentally maintain the imagined perspective in their mind for making a later spatial decision, and do not automatically move back to their sensorimotor perspective (Avraamides \& Ioannidou, 2005; Avraamides \& Kelly, 2008). Interestingly, there was no cost in RT in the response phase for incongruent trials, compared to congruent trials. If RT is not sensitive to perspective switching, it could be a better measure for VSPT instead of accuracy (i.e., error size), or a combined measure should be considered. Alternatively, we suggest that only the congruent trials in the $>90$ condition should be included as a measure of VSPT because the incongruent trials also contain a perspective switching component (when moving to the response board). Hence, the incongruent trials could provide a measure of perspective switching, a component that is part of VSPT, since VSPT requires moving from the sensorimotor information that one is holding to cognitive information one is mentalizing (May, 2004).

In trials with perspective changes smaller than $90^{\circ}$ (i.e., $<90$ condition), we found a strong correlation between the 
angular disparity (i.e., perspective changes) and the response time. This pattern was attributed in the literature to a mental rotation strategy (Hintzman et al., 1981; Shepard \& Metzler, 1971). This finding corresponds with Kozhevnikov and Hegarty's (2001) finding that trials with a perspective change smaller than $90^{\circ}$ did not involve VSPT, but other strategies like mental rotation. Kozhevnikov and Hegarty did not analyze RTs in their study and the finding was based on participants' self report. Given that participants employed a mental rotation strategy in the $<90$ condition, the congruent and incongruent trials are both indicators for mental rotation - the former of smaller angular disparity and the latter of larger angular disparity.

One limitation in JRD tasks that use visual maps (e.g., Kozhevnikov \& Hegarty, 2001) is that the objects in the layout are fixed, and as such, participants can memorize the layout, thus the accuracy can be affected by their memory skills. To prevent memorizing the layout, the dot colors were changed in each trial. In the first experiment, we divided the participants into two groups. Each group performed the DPT with a different arrangement of dots in the configuration. The results showed no differences between the two groups. Thus, we saw that performance in the DPT was not influenced by the arrangements of the configuration and that the effects we found were not restricted to a specific arrangement. Moreover, it seems that blocking or mixing the arrangements, thus making it harder for memorization, did not affect the results (as we found in Experiment 2). This finding strengthens the notion that participants did not memorize the dots' positions.

A few limitations of the current research should be discussed. First, we used two positions of the smiley (above and below the layout). The fixed positions might have affected the participants' performance since one position was aligned with a sensorimotor view of the layout and the other was in an opposite position. Thus, the smiley's position might have affected performance in addition to the perspective changes (larger or smaller than $90^{\circ}$ ). Future research could use different smiley positions that are not aligned and opposite to the viewer or use instead the locations of dots in the layout for determining the imagined position. Moreover, the smiley face was always turned to the viewer, so when it was below the layout it was not turned towards the layout. Creating an avatar that always faces the dots in the layout should be considered.

To conclude, analyzing accuracy and two distinct RTs, and examining the congruency of the perspectives in two phases of the task, deepened our understanding of the cognitive processes that are involved in a JRD task. Although the same set of stimuli was used, different mental transformation strategies were implemented in trials that involved perspective changes smaller than $90^{\circ}$, compared to trials that involved changes larger than $90^{\circ}$. Moreover, moving between an imagined perspective to a sensorimotor perspective in the response phase
(Avraamides \& Kelly, 2008) resulted in worse accuracy than staying in a congruent, mental perspective. Therefore, we suggest the perspectives in the layout and in the response circle be congruent to each other in order to eliminate the difficulty of perspective switching in JRD tasks.

VSPT is a complex process that in addition to mental transformation (e.g., Easton \& Sholl, 1995; Rieser, 1989; Shelton \& McNamara, 1997) also involves switching between a sensorimotor perspective (i.e., what we see through our eyes) to an imagined perspective (i.e., what we mentalize in our mind) (May, 1996, 2004). However, perspective switching is a component that so far has been neglected in VSPT research. In the current study we examined perspective switching within a single trial in a JRD task and suggest that further exploration of this component can enhance our understanding of VSPT.

Acknowledgements This work was supported by funding from the European Research Council under the European Union's Seventh Framework Programme (FP7/2007-2013)/ERC Grant Agreement No. 295644. We would like to thank Ronen Hershman, Naama Katzin, and Desiree Meloul for their professional and generous help. Finally, we thank three research assistants - Tal Feldman, Matanel Weissmann, and Ram Orr - for their help.

\section{References}

Avraamides, M. N., \& Ioannidou, L. M. (2005). Locating targets from imagined perspectives: Labeling vs. pointing. In B. G. Bara, L. Barsalou, \& M. Bucciarelli (Eds.), Proceedings of the $27^{\text {th }}$ Annual Meeting of the Cognitive Science Society, 175-180.

Avraamides, M. N., \& Kelly, J. W. (2008). Multiple systems of spatial memory and action. Cognitive Processing, 9(2), 93-106.

Barratt, E. S. (1953). An analysis of verbal reports of solving spatial problems as an aid in defining spatial factors. The Journal of Psychology, 36(1), 17-25.

Borich, G. D., \& Bauman, P. M. (1972). Convergent and discriminant validation of the French and Guilford-Zimmerman spatial orientation and spatial visualization factors. Educational and Psychological Measurement, 32(4), 1029-1033.

Brainard, D. H. (1997). The Psychophysics Toolbox. Spatial Vision, 10, 433-436.

Brown, W. (1932). Spatial integrations in a human maze. University of California Publications in Psychology, 5, 123-134.

Bryant, D. J., \& Tversky, B. (1999). Mental representations of perspective and spatial relations from diagrams and models. Journal of Experimental Psychology: Learning, Memory, and Cognition, 25(1), 137-156.

Carroll, J. B. (1993). Human cognitive abilities: A survey of factoranalytic studies. Cambridge: Cambridge University Press.

Dalecki, M., Hoffmann, U., \& Bock, O. (2012). Mental rotation of letters, body parts and complex scenes: Separate or common mechanisms? Human Movement Science, 31(5), 1151-1160.

de Vega, M., \& Rodrigo, M. J. (2001). Updating spatial layouts mediated by pointing and labelling under physical and imaginary rotation. European Journal of Cognitive Psychology, 13(3), 369-393.

Easton, R. D., \& Sholl, M. J. (1995). Object-array structure, frames of reference, and retrieval of spatial knowledge. Journal of Experimental Psychology: Learning, Memory, and Cognition, 21(2), 483-500. 
Eliot, J. (1987). Models of psychological space. New York, USA: Springer-Verlag.

Faul, F., Erdfelder, E., Lang, A.-G., \& Buchner, A. (2007). G*Power 3: A flexible statistical power analysis program for the social, behavioral, and biomedical sciences. Behavior Research Methods, 39, 175-191

Furlanetto, T., Becchio, C., Samson, D., \& Apperly, I. (2016). Altercentric interference in level 1 visual perspective taking reflects the ascription of mental states, not submentalizing. Journal of Experimental Psychology: Human Perception and Performance, 42(2), 158-163.

Goldberg, J., \& Meredith, W. (1975). A longitudinal study of spatial ability. Behavior Genetics, 5(2), 127-135.

Guilford, J. P., \& Zimmerman, W. S. (1948). The Guilford-Zimmerman Aptitude Survey. Journal of Applied Psychology, 32(1), 24-34.

Hegarty, M. (2010). Components of spatial intelligence. Psychology of Learning and Motivation, 52, 265-297.

Hegarty, M., \& Waller, D. (2004). A dissociation between mental rotation and perspective-taking spatial abilities. Intelligence, 32(2), 175-191.

Hintzman, D. L., O'Dell, C. S., \& Arndt, D. R. (1981). Orientation in cognitive maps. Cognitive Psychology, 13(2), 149-206.

JASP Team. (2018). JASP (Version 0.9) [Computer software]. https:// Jasp-stats.org/

Just, M. A., \& Carpenter, P. A. (1985). Cognitive coordinate systems: Accounts of mental rotation and individual differences in spatial ability. Psychological Review, 92(2), 137-172.

Kosslyn, S. M. (1994). Image and brain: The resolution of the imagery debate. Cambridge, MA: MIT Press.

Kozhevnikov, M., \& Hegarty, M. (2001). A dissociation between object manipulation spatial ability and spatial orientation ability. Memory \& Cognition, 29(5), 745-756.

Kyllonen, P. C., Lohman, D. F., \& Woltz, D. J. (1984). Componential modeling of alternative strategies for performing spatial tasks. Journal of Educational Psychology, 76(6), 1325-1345.

Loftus, G. R., \& Masson, M. E. (1994). Using confidence intervals in within-subject designs. Psychonomic Bulletin \& Review, 1(4), 476490.

Lohman, D. F. (1979). Spatial ability: A review and reanalysis of the correlational literature (No. TR-8). Stanford, CA: Stanford University California, School of Education.

Lohman, D. F. (1988). Spatial abilities as traits, processes, and knowledge. In R. J. Sternberg (Ed.), Advances in the psychology of human intelligence (Vol. 4, pp. 181-248). Hillsdale, NJ: Lawrence Erlbaum Associates.

Lynch, K. (1960). The image of the city (Vol. 11). Cambridge, Massachusetts: MIT press.

May, M. (1996). Cognitive and embodied modes of spatial imagery. Psychologische Beitrage, 38, 418-434.
May, M. (2004). Imaginal perspective switches in remembered environments: Transformation versus interference accounts. Cognitive Psychology, 48(2), 163-206.

Mou, W., McNamara, T. P., Valiquette, C. M., \& Rump, B. (2004). Allocentric and egocentric updating of spatial memories. Journal of Experimental Psychology: Learning, Memory, and Cognition, 30(1), 142-157.

Pelli, D. G. (1997). The videotoolbox software for visual psychophysics: Transforming numbers into movies. Spatial Vision, 10, 437-442.

Piaget, J., \& Inhelder, B. (1956). The child's concept of space. London: Routledge \& Paul.

Piaget, J., \& Inhelder, B. (1966/1971). Mental imagery in the child: A study of the development of imaginal representation (PA Chilton, Trans.). New York: Basic Books.

Price, L., \& Eliot, J. (1975). Convergent and discriminant validities of two sets of measures of spatial orientation and visualization. Educational and Psychological Measurement, 35(4), 975-977.

Rieser, J. J. (1989). Access to knowledge of spatial structure at novel points of observation. Journal of Experimental Psychology: Learning, Memory, and Cognition, 15(6), 1157-1165.

Roff, M. (1952). A factorial study of tests in the perceptual area (Psychometric Monograph, No. 8). Richmond, VA: Psychometric Corporation.

Shelton, A. L., \& McNamara, T. P. (1997). Multiple views of spatial memory. Psychonomic Bulletin \& Review, 4(1), 102-106.

Shepard, R. N., \& Cooper, L. A. (1982). Mental images and their transformations. Cambridge, MA: The MIT Press.

Shepard, R. N., \& Metzler, J. (1971). Mental rotation of threedimensional objects. Science, 171(3972), 701-703.

Surtees, A., Apperly, I., \& Samson, D. (2013). Similarities and differences in visual and spatial perspective-taking processes. Cognition, $129(2), 426-438$.

StatSoft, Inc. (2014). STATISTICA (Version 12) [Data analysis software system]. www.statsoft.com.

Wolbers, T., \& Hegarty, M. (2010). What determines our navigational abilities? Trends in Cognitive Sciences, 14(3), 138-146.

Wraga, M., Shephard, J. M., Church, J. A., Inati, S., \& Kosslyn, S. M. (2005). Imagined rotations of self versus objects: an fMRI study. Neuropsychologia, 43(9), 1351-1361.

Zacks, J. M., \& Michelon, P. (2005). Transformations of visuospatial images. Behavioral and Cognitive Neuroscience Reviews, 4(2), 96-118.

Publisher's note Springer Nature remains neutral with regard to jurisdictional claims in published maps and institutional affiliations. 